Revue interdisciplinaire sur la Grèce archaïque

\title{
Danses éducatives et jeux érotiques : de Platon à l'Odyssée
}

Educational Dances and Erotic Games: From Plato to the Odyssey

\section{Claude Calame}

\section{OpenEdition}

\section{Journals}

Édition électronique

URL : https://journals.openedition.org/gaia/1965

DOI : 10.4000/gaia.1965

ISSN : 2275-4776

Éditeur

UGA Éditions/Université Grenoble Alpes

Édition imprimée

ISBN : 978-2-37747-292-5

ISSN : 1287-3349

Référence électronique

Claude Calame, «Danses éducatives et jeux érotiques : de Platon à l'Odyssée », Gaia [En ligne], 24 |

2021, mis en ligne le 31 mai 2021, consulté le 09 décembre 2021. URL : http://

journals.openedition.org/gaia/1965; DOI : https://doi.org/10.4000/gaia.1965

Ce document a été généré automatiquement le 9 décembre 2021.

Gaia. Revue interdisciplinaire sur la Grèce archaïque 


\title{
Danses éducatives et jeux érotiques : de Platon à l'Odyssée
}

\author{
Educational Dances and Erotic Games: From Plato to the Odyssey
}

\author{
Claude Calame
}

1 «Éros metteur en scène des corps »? tel est en tout cas l'intitulé de l'étude qu'Ezio Pellizer consacre à une interrogation collective sur les corps en jeu depuis l'Antiquité gréco-romaine. Et d'affirmer en particulier : "L'image corporelle d'Éros est construite selon le modèle d'un pàis, un jeune qui n'est pas complètement sorti de l'âge des jeux, d'une façon telle que cette image éphébique (ou pré-éphébique, d'adolescent) semble tendre avec facilité à une multiplication. » $(2010,130)$

2 Or il est un fragment de poésie érotique, transmis sous le nom du poète Spartiate Alcman, à établir explicitement le lien entre la qualité de $\pi \alpha \bar{\imath} \varsigma$ et le jeu comme pratique du $\pi \alpha{ }^{\prime} \zeta \varepsilon v v^{1}$ :

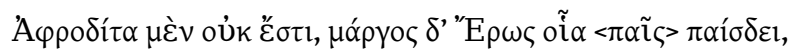

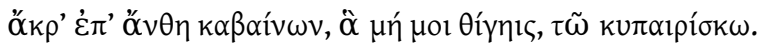

Ce n'est pas Aphrodite, mais Éros l'insensé qui s'amuse comme un enfant

À voleter sur la corolle des fleurs - ne va pas me les toucher ! - du souchet.

3 En dépit de l'erreur d'haplographie commise par le scribe, le schéma métrique de ces deux vers cités par Héphestion, comme exemple de ce que les métriciens modernes identifient comme hexamètre crétique catalectique, est significatif. Il donne un sens particulier au jeu de mot étymologisant qu'il formule autour du terme $\pi \alpha \bar{i} \varsigma$; ce terme désigne le jeune garçon, mais parfois aussi la jeune fille, et « jouer » c'est se comporter en jeune adolescent.

4 Mais de quel « jeu » s'agit-il ? La piste sémantique à suivre est indiquée par le poème même dont ces deux vers sont extraits. En effet, au-delà de sa technicité, l'évocation du schéma métrique qui rythme ces deux vers composés par Alcman nous rappelle qu'ils proviennent d'un poème chanté et dansé sur un accompagnement musical ; c'est donc 
un poème non pas «lyrique » mais appartenant au grand genre du mélos, avec ses différentes formes essentiellement chorales ${ }^{2}$.

Quand il s'agit d'art choral la référence est obligée à Platon, par-delà toute la production mélique qui a marqué le $\mathrm{vI}^{\mathrm{e}}$ et le $\mathrm{v}^{\mathrm{e}}$ siècles; cela en raison de l'attention qu'il porte, surtout dans les Lois, à la place à réserver dans la cité idéale à la xopzía. Au terme du livre II, les trois interlocuteurs du dialogue en reviennent à la question de l'éducation rendue nécessaire par les mouvements incontrôlés et les cris désordonnés de tout être vivant avant d'avoir atteint l'âge adulte; la référence à ce que dit la légende sur Dionysos à qui sa marâtre Héra aurait fait don du vin pour le mettre dans un état de folie. Face au Crétois Clinias l'étranger d'Athènes réaffirme les deux piliers traditionnels de l'éducation comprise comme $\pi \alpha i ́ \delta \varepsilon v \sigma ı \varsigma$ : la musique et la gymnastique

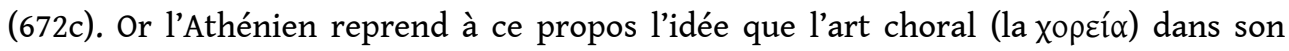
ensemble c'est l'éducation ( $\left.\pi \alpha \alpha_{i}^{\delta} \delta \varepsilon \sigma ı \zeta\right)$ dans son ensemble; et cela dans la mesure où l'art choral permet de maîtriser à la fois voix et gestes $(672 \mathrm{e})^{3}$.

6 En effet, de ce point de vue de l'art choral, il convient de distinguer entre d'une part les rythmes et les harmonies qui relèvent de la voix et d'autre part les mouvements du corps qui pour le rythme sont en accord avec la voix dont le mouvement propre est la mélodie, le $\mu$ ćloc (673a)! Est ainsi entraînée d'une part l'âme soumise par le

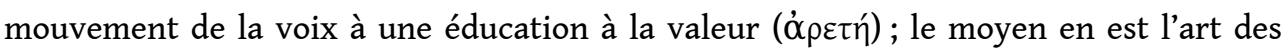

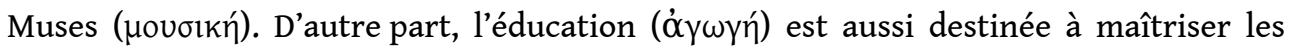

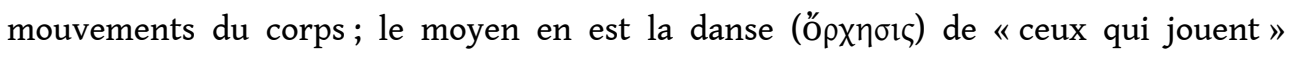
( $\pi \alpha \zeta \zeta o ́ v \tau \omega v)$ dans un mouvement conduisant à la même vertu par un art technique qui a pour nom la gymnastique ( $ү u \mu v \alpha \sigma \tau i k \eta ́)$.

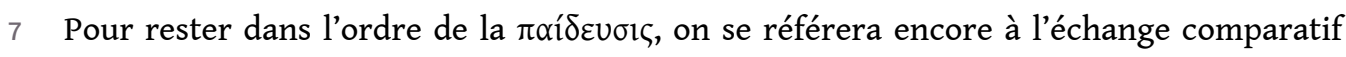

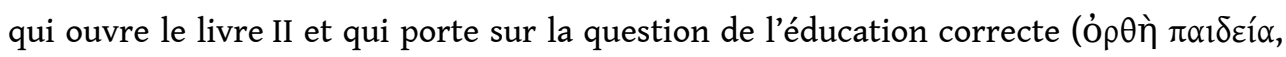
653a). Quant à la maîtrise des plaisirs et des afflictions essentielles sont pour les hommes adultes les célébrations festives qui sont accordées par les dieux sous l'égide des Muses, d'Apollon Musagète et de Dionysos. Et pour les enfants qui gambadent et bondissent en jouant ( $\pi \rho \circ \sigma \pi \alpha$ (́) $о v \tau \alpha)$ et en émettant toutes sortes de sons s'impose l'ordre impliqué par le rythme et par l'harmonie. Ainsi, à l'issue d'une longue affirmation initiale face au Crétois Clinias, l'Athénien le déclare d'emblée et

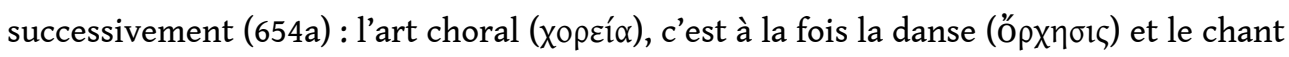
$\left(\grave{\omega} \delta \eta^{4}\right)^{4}$; l'éducation première est le fait des Muses et d'Apollon; et finalement qui ne

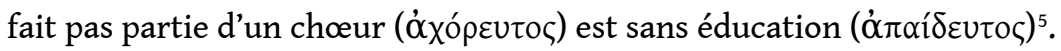

8 En définitive, le $\pi \alpha$ íł $\varepsilon v$ est donc assimilé au rythme chorégraphique du corps, tout en étant animé par la voix référée à l'âme.

9 Mais de Platon revenons aux poèmes homériques que notre tradition ne cesse de

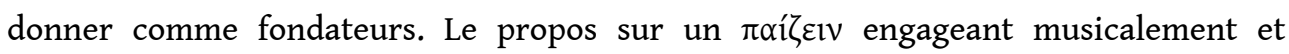
rythmiquement le corps et l'âme ne saurait être mieux illustré que par deux mises en scène chorales insérées, sur le mode poétique et aédique, dans le récit de l'Odyssée.

On se rappelle la réception que le roi Alcinoos organise pour Ulysse en son palais de Phéacie. Après une première intervention chantée de l'aède Démodocos, la rencontre est marquée par des concours gymniques (VIII, 246-265). Puis, non sans avoir vanté les pratiques gymniques et musicales des jeunes Phéaciens, le roi Alcinoos s'adresse aux meilleurs danseurs. Il les invite à accompagner de leurs pas de danse le chant de Démodocos, appelé ici à intervenir une seconde fois. Il s'agit d'impressionner l'hôte 
Ulysse qui pourra emporter en son île d'origine le souvenir des pratiques où les

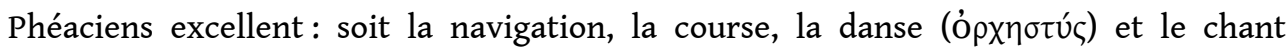

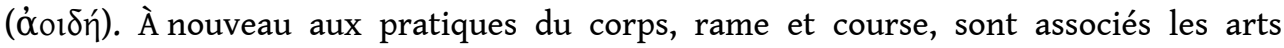

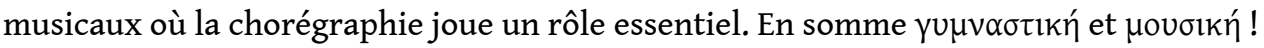

Pour accompagner l'aède qui lui-même s'accompagne sur la phorminx, les jeunes gens s'organisent en une troupe qui bat de ses pieds l'aire chorale aménagée par neuf arbitres; par deux fois (vers 262 et 269) cette aire chorale est désignée par le terme xopóc, dans une acception spatiale que le mot assume parfois, une aire chorale qui est

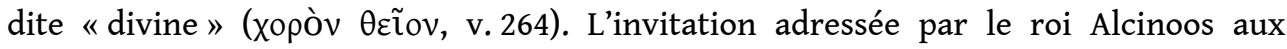

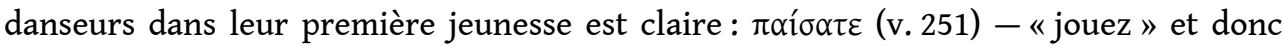
«dansez! ». Et ces pas de danse, sous les yeux d'Ulysse admiratif, vont scander le récit que Démodocos va faire, en s'accompagnant dûment sur la phorminx, des folles amours d'Arès et d'Aphrodite au détriment d'Hépaïstos.

Par ailleurs, c'est l'usage de ce même verbe $\pi \alpha$ íł̌clv qui caractérise la scène non moins fameuse où Nausicaa, accompagnée de ses suivantes ( $\dot{\alpha} \mu \varphi i ́ r o \lambda o r)$, se dirige vers les eaux d'un fleuve pour une lessive fortement marquée par Éros (VI, 85-109). C'est à cette fameuse scène qu'assiste Ulysse, à peine rejeté sur l'île des Phéaciens et caché nu dans un sous-bois. On se souvient qu'au début du chant VI, Athéna intervient auprès de Nausicaa, dans le palais de son père Alcinoos. La déesse invite la gracieuse jeune fille à laver à la rivière les beaux vêtements qui éveilleront l'intérêt de prétendants pour la conduire au mariage. Nausicaa approche son père sous prétexte de laver en particulier les vêtements que portent ses trois plus jeunes fils, constamment désireux de fréquenter l'aire chorale ( $\dot{\varepsilon} \zeta$ Xopóv, v. 65). Le père comprend les enjeux de la demande de sa fille et, le corps dûment oint et accompagnée de ses suivantes ( $\dot{\alpha} \mu \varphi i ́ r o \lambda o 1, ~ v . ~ 84)$, Nausicaa dirige vers les eaux d'un fleuve le char chargé des vêtements destinés à une lessive chargée de sens.

Puis, en attendant qu'étendu sur la plage sèche au soleil le linge lavé à l'eau claire du fleuve, les jeunes filles se lavent, s'oignent d'huile et se restaurent. Puis rejetant de leur

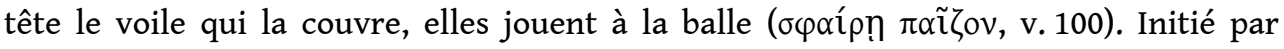

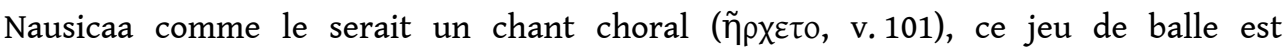
effectivement désigné comme $\mu \mathrm{o} \lambda \pi \eta ́$ (v. 101) ; l'usage de ce terme qui renvoie comme celui de mélos à un chant dansé sur un accompagnement musical fait bien correspondre ce jeu à un chant choral ${ }^{6}$ ! Puis dans la fonction de chorège que lui attribue le fait de " commencer» le chant, Nausicaa est comparée à Artémis quand elle évolue sur le Taygète ou l'Érymanthe au milieu des nymphes agrestes ${ }^{7}$, ces filles (кoũ $\rho \alpha_{1}$ ) de Zeus dansent avec la déesse : $\pi \alpha_{i}^{\prime} \zeta$ ovor (v. 106) ! Est ainsi évoquée une autre scène musicale, insérée dans le récit odysséen.

Mère d'Artémis, Létô se réjouit de cette scène chorale, de même que, par exemple, son fils Apollon, dans l'hymne homérique qui lui est consacré, se laisse charmer et se réjouit des joutes de pugilat ainsi que des chants et des danses chorales des Ioniens et des Ioniennes rassemblés en son sanctuaire de Délos pour le célébrer: à nouveau gymnastique et arts des Muses vont de pair ${ }^{8}$. Les nymphes sont belles : mais parmi elles

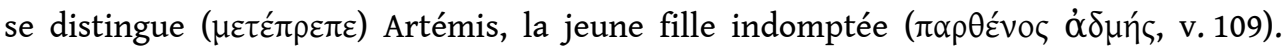
Ainsi en va-t-il également, par exemple, d'Hagésichora, la chorège au visage d'argent chantée par un groupe choral de jeunes filles dans le "premier " parthénée composé par le poète Alcman, dont on a cité deux vers en guise d'introduction. La jeune femme se distingue comme un «destrier vigoureux, victorieux aux jeux, au galop 
retentissant » au milieu d'une troupe de cavales, suscitant le désir des jeunes choreutes comme le montre la suite du poème ${ }^{9}$. Une fois encore l'attention est focalisée sur le mouvement des jambes et des pieds.

S'il n'est pas explicitement convoqué dans la scène homérique, c'est évidemment Éros qui anime les pas de danse des jeunes filles et de leur chorège, suscitant le désir de qui les entend et les regarde. Rappelons encore la scène qui fait suite à la lessive dansante des suivantes de Nausicaa et de leur belle chorège. Athéna fournit à Ulysse l'occasion de sortir du bosquet d'où, dissimulé, il avait assisté à la scène de l'érotique jeu de balle dansant ; une suivante manque la balle lancée par Nausicaa et les cris des jeunes files suscitent la réflexion habituelle d'Ulysse abordant sur une terre inconnue: suis-je confronté à de sauvages mortelles ou à des personnes hospitalières? Sont-elles des nymphes ou appartiennent-elles au genre des hommes au langage articulé ? Tout en ayant soin de couvrir ses parties, Ulysse nu sort alors du bois: les jeunes filles s'enfuient à cette vue sauf Nausicaa, retenue par Athéna, et Ulysse adresse à la jeune fille «aux beaux yeux » (v. 142) de douces paroles. Scène érotisée s'il en est de même que les mots qu'Ulysse adresse à la jeune femme: comparaison avec Artémis

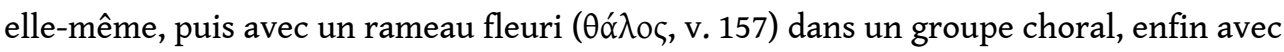
la jeune pousse (véov ع̌ $\rho v o \varsigma$, v. 163) du palmier vu à Délos auprès de l'autel consacré à Apollon $^{10}$. Et finalement, après un bain, le corps dûment oint, vêtu des habits choisis par Nausicaa, rendu plus fort encore par Athéna, Ulysse resplendissant de beauté et de

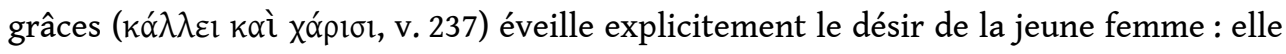
exprime le souhait que cet homme divin puisse devenir son époux (v. 244)!

Le jeu d'Éros, c'est la danse d'Éros ; et la danse renvoie au poème chanté qui convoque, de manière performative, l'être divin et qui en est la réalisation chorégraphique.

Avec Ezio Pellizer on reviendra donc en conclusion à la poésie mélique, et en particulier aux vers adressés par le chant d'Anacréon à la fameuse « pouliche de Thrace » qui jette vers le je poétique un regard dont on sait qu'il est le véhicule de la puissance d'Éros ${ }^{11}$. Elle se livre à un jeu d'esquive typique du rapport érotique asymétrique qui est par ailleurs illustré par la relation entre la jeune Nausicaa et Ulysse l'adulte. Elle est la pouliche de Thrace que le poète souhaite juguler et guider pour la soumettre à une course toute métaphorique avant d'en être l'habile jockey; elle est la pouliche de Thrace qui «maintenant» (võv $\delta \grave{\varepsilon}$, v. 5 en écho au déictique $\delta \eta ́$ au début du chant: espace et temps de la performance chantée du poème!) joue ( $\pi \alpha \hat{\alpha} \zeta \varepsilon 1 \zeta)$ en bondissant d'un pas léger dans les prés. Non seulement cette indication d'ordre paysager nous reconduit aux espaces de déploiement et d'assouvissement du désir érotique : tendres prairies, souvent émaillées de fleurs aux couleurs et surtout aux parfums séducteurs ; mais surtout le jeu est associé au pas bondissant des jeunes gens comme des jeunes filles qui n'ont pas encore reçu l'éducation chorale prescrite par Platon. 


\section{BIBLIOGRAPHIE}

BETTINI Maurizio \& PELLIZER Ezio, Il mito di Narciso. Immagini e racconti dalla Grecia ad oggi, Turin, Einaudi, 2003.

CALAME Claude, «Identifications génériques entre marques discursives et pratiques énonciatives : pragmatique des genres "lyriques" ", dans R. Baroni \& M. Macé (éd.), Le savoir des genres, Rennes, La Licorne, 2006, p. 35-55.

CALAME Claude, « Le regard amoureux. Physiologie des sens, langue poétique et pragmatique du chant érotique en Grèce ancienne ", dans M.-L. Gélard (éd.), Corps sensibles. Usages et langages des sens, Nancy, Presses universitaires de Lorraine, 2013, p. 53-74.

CALAME Claude, « Les pratiques chorales dans les Lois de Platon : une éducation à caractère initiatique? ", dans L. Brisson \& O. Renaut (éd.), Érotique et politique chez Platon. Erôs, genre et sexualité dans la cité platonicienne, Sankt Augustin, Academia, 2017, p. 151-167.

CALAME Claude, Les chœurs de jeunes filles en Grèce ancienne. Morphologie, fonction religieuse et sociale (Les parthénées d'Alcman), Paris, Les Belles Lettres, 2019a.

CALAME Claude, « Métaphores végétales de la croissance, sanctuaires paysagers, pratiques cultuelles : parcours initiatiques pour les jeunes filles ", dans A. Gartziou-Tatti \& A. Zografou (éd.), Des dieux et des plantes. Monde végétal et religion en Grèce ancienne, Kernos, suppl. 34, Liège, Presses universitaires de Liège, 2019b, p. 59-68.

GARELLI Marie Hélène \& VISA-ONDARÇUHU Valérie (éd.), Corps en jeu de l'Antiquité à nos jours, Rennes, Presses universitaires de Rennes, 2010.

KOWALZIG Barbara, « Broken Rythms in Plato's Laws: Materializing Social Time in the Chorus », dans A.-E. Peponi (éd.), Performance and Culture in Plato's Laws, Cambridge, Cambridge University Press, 2013, p. 171-211.

NAGY Gregory, Pindar's Homer. The Lyric Possession of an Epic Past, Baltimore / Londres, The Johns Hopkins University Press, 1990.

PELLIZER Ezio, «Éros metteur en scène des corps », dans M. H. Garelli \& V. Visa-Ondarçuhu (éd.), Corps en jeu de l'Antiquité à nos jours, Rennes, Presses universitaires de Rennes, 2010, p. 125-134.

PEPONI Anastasia-Erasmia, «Choreia and Aesthetics in the Homeric Hymn to Apollo: The Performance of the Delian Maidens (Lines 156-64) », ClAnt, 28, 2009, p. 39-70.

ROSENMEYER Patricia A., « Girls at Play in Early Greek Poetry », AJPh, 125, 2004, p. 163-178.

\section{NOTES}

1. Alcman, fr. 58 Page = 147 Calame, cité par Héphestion, Enchiridion, 13,6 (p. 42, 13 ss.

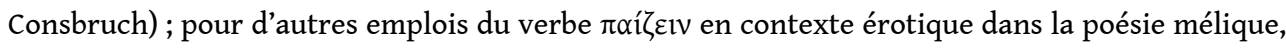
voir par exemple Anacréon, fr. 358 Page, avec la bonne étude de Rosenmeyer (2004).

2. Quant aux confusions entraînées par l'emploi du concept moderne de «lyrique " pour la désignation des différentes formes de la poésie mélique, voir notamment Calame (2006).

3. On verra à ce propos en particulier l'étude de Kowalzig (2013) ainsi que les nombreuses références données dans Calame (2017). 
4. On se souviendra ici du jeu de mot étymologisant suggéré dans ce même passage des Lois (654a)

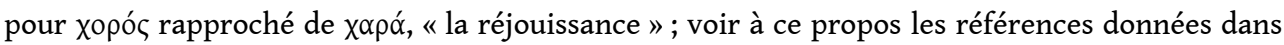
Calame (2019a, 62, note 3).

5. Davantage à ce propos dans l'étude de Calame (2017).

6. Quant au sens de ce mot on verra les références données dans Calame (2019a, 167-168, avec la note 239).

7. Assumer le prélude du chant indique la fonction de conduite qu'y assume le ou la chorège : cf. Calame (2019a, 108-115), avec les remarques complémentaire de Nagy (1990, 344-354) ; quant aux paysages dans lesquels évoluent les nymphes, voir par exemple Bettini \& Pellizer (2003, 117-121). 8. Hymne homérique à Apollon, 146-155, dans une scène souvent commentée en ce qu'elle précède l'intervention du chœur des jeunes Déliades; voir par exemple à ce propos l'étude de Peponi (2009).

9. Alcman, fr. 1, 43-49 Page = 3, 43-49 Calame.

10. Voir l'étude de Calame (2019b) qui se fonde en particulier sur le travail de thèse d'Alessandro Buccheri (à paraître).

11. Anacréon fr. 417 Page ; pour le regard comme véhicule du désir érotique, voir Pellizer (2010, 129-131), ainsi que Calame (2013).

\section{RÉSUMÉS}

Dans une contribution récente, Ezio Pellizer s'interrogeait sur les relations entre Eros, le pais et la mise en scène du corps. Or dans les Lois de Platon l'Athénien reprend à ce propos l'idée que l'art

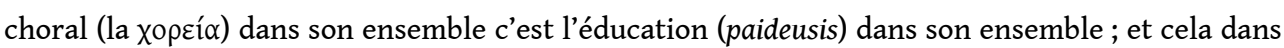
la mesure où l'art choral permet de maîtriser à la fois voix et gestes. De là les deux fondements de l'éducation destinée aux paides : la musique et la gymnastique. À travers quelques vers de poètes dits «lyriques» et une scène célèbre de l'odyssée, on aimerait montrer les rapports que les pratiques chorales tissent, pour les jeunes gens comme pour les jeunes filles, entre la danse conçue comme paizein, l'éducation chorale, et les sentiments animés par Eros, lui-même un pais.

In a recent contribution, Ezio Pellizer mused upon the relationship between Eros, the pais and the staging of the body. In Plato's Laws, the Athenian takes on the idea that the choral art

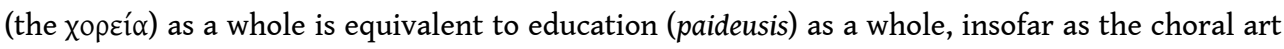
allows the mastery of both voice and gestures. Hence, the two bases of the education for the paides were music and gymnastics. Based on an exploration of a few lines by poets called "lyrics" and a famous scene from the Odyssey, this work seeks to point out the relationships established by choral practices for the young man and for the young woman, in relation to dance as paizein, choral education, and the feelings raised by Eros, himself a pais.

\section{INDEX}

Mots-clés : choreia, éducation, Eros, jeunes filles, poésie mélique

Keywords : choreia, education, Eros, young women, melic poetry 
AUTEUR

\section{CLAUDE CALAME}

École des hautes études en sciences sociales (EHESS), Paris

claude.calame@unil.ch 